running catgut suture in two stages. The patient died suddenly on the fourth day from secondary hæmorrhage, which, as the autopsy showed, came from the wounded vein, from which the ligature bad become loosened. The closure of the bladder wound was found perfect.

The foregoing list is by no means complete, but the number of cases it includes - sixty-seven cases among forty-one operators-is sufficiently large to show that the accident is by no means infrequent.

Some surgeons manifest a reluctance to making known the various mishaps which occur in their operative work, in the belief, perhaps, that a knowledge of them will injuriously affect their reputation with their colleagues. I consider this an error of judgment. Surgeons should be honest as well as skilful, and their integrity is quite as likely to receive recognition as their dexterity, and certainly is of more value.

Considering the conditions under which bladder injuries may happen during laparotomy, it is not discreditable to any surgeon to meet with them, for they may not be due to any carelessness or lack of skill on his part. In many of the foregoing cases no possible degree of diligence could have averted the accident. Adhesions of the peritoneal surface of the elongated bladder to that of the anterior abdominal wall frequently cannot be known in advance, and their existence is only demonstrable after the viscus has been opened. The use of the catheter as a diagnostic means is not always available, because the compression of the bladder against the pubis may prevent the introduction of the instrument beyond that point. Certainly, however, this should always be attempted in any case of suspected difficulty, and would seem to be even a proper and unobjectionable routine method.

Another useful precaution is to avoid prolonging the abdominal incision far down towards the pubic bone until the opening into the peritoneum has permitted the relations of the bladder to be ascertained.

The mortality of the cases in which the bladder has been wounded is large, namely about 30 jei cant.; but this is due to the complicated and serious character of the cases in which the accident has occurred, the consequently increased length of the operation and the greater danger from shock, rather than to the mere vesical injury. The latter, indeed, does not seem of itself to be very important as influencing the recovery of the patient; but, notwithstanding this fact, a urinary fistula adds greatly to her discomfort, and, occurring under such circumstances, must be productive of chagrin and annoyance to the surgeon.

Inasmuch as the bladder is recognizable with more difficulty when empty than when full, it would be better, in cases presenting doubtful fea- tures, to commence the operation with the viscus wholly or partly distended. When its position has become known, after the completion of the abdominal incision, it may be emptied by an assistant.

Treatment.--When it is known at the time of operation that the bladder has been cut or torn, the opening should be at once closed with a continuous suture of catgut or fine silk, applied so as to invert the edges of the wound and bring together the peritoneal surfaces. A permanent catheter ought to be used during the first two days. After the expiration of that time its constant use is usually unnecessary; and if the wound has been small-less than one inch in lengththe instrument may be subsequently dispensed with. If, however, the wound has been large-exceeding two or three inches-the bladder ought to be artificially emptied as often as every three hours during three or four days additional. In all cases the catheter should be used so long as the urine contains blood.

In the case in which urine appears through the abdominal wound subsequently to the operation, at a time and under circumstances which might make it dangerous or inexpedient to reach the seat of the vesical injury, the catheter ought to be used either continuously or at short intervals, for the purpose of lessening the amount of urine which escapes through the fistula, and thus aid in the closure of the latter. If, however, the fistulous opening should show no disposition to close after two or three months, the edges ought to be freshened to the depth of half an inch or more and stitched together.

In exceptional instances it may be expedient to affix the wounded edges of the bladder within those of the abdominal incision, in the manner detailed by Thomas and others; but as this method must interfere, to some extent, with the subsequent contractility of the bladder, it is not to be commended as a usual practice. The suturing and "dropping" of the vesical wound is the better method.

\section{THE TEETH AND ORAL CAVITY OF PREGNAN'T WOMEN.}

Read in the Section of Oral and Dental Surgery, at the Fortieth An nual Meeting of the American Medical Association, June, Is8g.

$$
\begin{gathered}
\text { BY JOHN S. MARSHALL, M.D., } \\
\text { of chicago. }
\end{gathered}
$$

From time almost immemorial, pregnancy has been recognized as having a deleterious effect upon the teeth; and this opinion has become so general that it has been crystallized into the familiar adage--" for every child a tooth." All writers upon obstetrics and the care of pregnant women, recognize the fact that during the periods of gestation and lactation women are more liable to suffer from diseases of the teeth and neu- 
ralgia of the facial region, than at other times; but the causes, prevention or mitigation of these diseases has received but little attention; in fact, it seems to be considered by most of them that they are among the inevitable consequences of gestation and that little can be done to relieve them.

With this view I do not fully agree, for I believe much can be done to prevent or relieve the suffering incident to caries and the loss of the teeth, and the neuralgic conditions of the face and mouth so common at this period. But in order to appreciate the causes and the treatment of these disorders, we must have in mind the changed conditions of the organism. The pregnant state is characterized by many and varied changes in the general system and in special organs, the nuost marked of which are those of the uterus, the genitals, and mammary glands, but which it is not necessary for us to take time to describe, as they are generally well understood. The changes which bear the closest relationship to our subject, are those of nutrition affecting the condition of the blood, the bones, the teeth and the excretions and secretions.

Pregnancy usually exercises a favorable influence upon nutrition; after the third or fourth month the appetite improves, adipose tissue is laid on and the body weight is progressively increased, so that at the completion of gestation, it is about one-thirteenth greater than before. ${ }^{1}$ Many times, however, the reverse of this is met with; nutrition is so disturbed as to cause considerabie emaciation and general debility.

The blood is increased in volume during the second half of gestation, as was demonstrated by Spiegelberg and Gescheidlen ${ }^{2}$ in experiments upon pregnant bitches. Authorities, however, are not agreed as to changes in the character of the blood. Andral, ${ }^{3}$ Nasse, ${ }^{4}$ Meyer $^{5}$ and others claim that the watery elements and the white corpuscles are increased and the red corpuscles diminished. Ingerslev ${ }^{6}$ could detect no diminution in the number of the red corpuscles; while on the other hand Fehling found the hæmoglobin and the red corpuscles increased. Hypertrophy of the left ventricle, of temporary character, sometimes occurs as the result of increased labor thrown upon the heart consequent upon the augmentation of the blood mass. This was first made known by Larcher ${ }^{8}$ in 1828 . Venous congestion, varices, and swelling of the lower extremities and arterial hyperæmia of the upper half of the body is a frequent accompaniment of pregnancy. The causes of these conditions are not fully understood, but were attributed by $\mathrm{De}$ Cristoforis ${ }^{9}$ to pressure of

\footnotetext{
I Hirst's American System of Obstetrics, p, 353

Ibid., p. 345

Animals de Chymie et de Physique, Juellet, $\varepsilon_{42}$

4 Archives of Gyn. Bd. $x, p .338$

"Untersuchungen uber die Zeianderungen des Blutes in der Schroangerschaft."

Centralb. f. Gyn., I879, p. 635

Archives of Gyn,, Bd. xxvili, Heft 3, p. 454.
}

the gravid uterus upon the iliac veins preventing the return of blood to the inferior vena cava, and pressure upon the descending aorta, thus obstructing the normal flow of blood to the lower extremities and causing arterial hyperæmia of the upper portion of the body.

Changes in the constituent elements of the bones are of common occurrence as a result of malnutrition. Dalton ${ }^{10}$ says: "Next to the chloride of sodium, the phosphate of calcium is considered the most important mineral ingredient of the body. It is met with universally in every tissue and every fluid" - and "whenever the nutrition of the bone during life is interfered with from any pathological cause, so that its phosphate of calcium becomes deficient in amount, a softening of the osseous tissue is the consequence, by which the bone yields to external pressure and becomes more or less distorted." Softening or decalcification of the bones undoubtedly takes place in a limited extent in many cases of pregnancy. In fractures occurring during gestation, union is often delayed, sometimes until after delivery. Padieu ${ }^{11}$ describes a case in which fractures of the tibia and fibula occurred nine days after the suppression of the menses, and in which union was delayed until the end of gestation. The process of union began ten days after delivery and was completed at the end of a month. On the other hand, the bones of the extremities frequently increase in length, and osteophytes are sometimes formed both intraand extra-cranial.

According to Förster, ${ }^{12}$ in a little more than one-half of the cases of pregnancy examined after death, there were found deposited upon the inner surface of the skull, thin lamellæ of a bone-like formation, composed of phosphate and carbonate of calcium, and measuring from $1 / 6$ to $1 / 2$ of a line in thickness. These formations are most commonly found upon the frontal and parietal bones, in the neighborhood of the sulcus falciformis and the arteria meningia media, and usually occur after the third month. These formations are not, however, exclusively an accompaniment of pregnancy, for they are frequently found associated with tuberculosis, and might therefore be classed as effects of insufficient nutrition, rather than of over-nutrition.

The pelvis, though looked upon at all other times as a comparatively solid framework, frequently becomes relaxed in its articulations during pregnancy so that the sacro-iliac and pubic joints become movable. Luschka ${ }^{13}$ demonstrated that these articulations were true joints. Lenoir ${ }^{14}$ found in twenty-two pregnant women between the ages of 18 and 35, movement of the sacro-iliac and

\footnotetext{
${ }^{8}$ Hirst's Amer. Syst, of Obstetrics, p. 346 .

8 Hirst

Io Daiton's Physiology, fifth edition, p. 58

Ir Journal de Médicine et de Chirurgie pratique, 1887

12 Handbuch der patholog. Anat., Vol. ii, p. 945.

Is Die Anatomie des Menschlicken, Bukens, Tubingen, 3864 .

14 Hirst's American System of Obstetrics, p. $34 \mathrm{I}$
} 
pubic articulations. Occasionally such movement is so great as to make locomotion difficult or impossible.

Lawson Tait $^{15}$ is authority for the statement that the thyroid g!and frequently increases in size during pregnancy, and that he noted in districts where goitre was endemic, and in women in whom there existed the predisposition, pregnancy may produce a temporary form of the disease or furnish the exciting cause for the development of the permanent disease.

The spleen, in all probability, is increased in size during gestation..$^{10}$ The functions of the kidneys are also heightened and in consequence of this they become somewhat larger. ${ }^{17}$ The urine undergoes change both in quantity and quality ; the aqueous elements are increased in consequence of the augmentation in the blood mass and the high arterial tension. Winckel found no change in the amount of urea, sodium chloride, sulphates and phosphates daily excreted. Chalvet and Barlemont, on the other hand, found the chlorides increased in quantity and the phosphates, sulphates, urea, uric acid, creatin and creatinin diminished in amount. Lehmann and Donne ${ }^{\text {ls }}$ have suggested that the cause of this deficiency was due to the extra demand for these substances in the development of the foetus. Glucose and albumen are not infrequently present in the urine during pregnancy. An increase in the salizary secretions is often a noticeable symptom. I'tyalism, when present, manifests itself early and usu. ally disappears spontaneously between the third and fourth months. It occasionally persists, however, in an aggravated form during the entire period of gestation and even for several weeks after, and the amount secreted may be so great as to endanger the life of the patient. The qualitative changes in the saliva during pregnancy are sometimes quite marked. The water is increased, while the organic and inorganic elements are diminished. Schramm reported one case in which the ptyalin was entirely absent. In those cases in which excessive ptyalism is manifested the buccal mucous membrane is more or less inflamed, the parotid, submaxillary and sublingual glands are swollen and tender, and quite painful when their functions are especially excited. Fetor is not present, and the absence of this symptom distinguishes it from mercurial ptyalism. The cause of this disorder is in all probability a reflex neurosis, and it frequently reappears in successive pregnancies. Gingivitis is another common accompaniment of pregnancy, and is often present when there is no indication of salivation. Pinard is of the opinion that it is more common among multiparæ than primiparæ. This opinion is based upon seventy-five cases, of whom forty-three were

\footnotetext{
is Obst. Jour. of Great Britain and Ireland, I875.

16 Wood's Handbook Medical Sciences, Tol. vi, p. 5

1) Hirst's American System of Obstetrics, Vol, $i, p .3 .34$.

s 8 Ibid., Vol. i, p. 354
}

multiparæ and thirty-two primiparæ. In the former the disorder was present thirty-one times, in the latter fourteen times. Gingivitis is characterized by redness and tumefaction of the gums, and a tendency to bleed on slight pressure or friction, as in mastication or the use of the tooth-brush; while the secretions of the mucous gland are often decidedly acid in reaction, as may be easily proved by litmus paper. Treatment should consist of saline cathartics and astringent and antacid mouthwashes, and thorough cleanliness of the mouth and teeth.

Phagedenic pericementitis is occasionally present during pregnancy, and right here let me call your attention to the fact that this disease is often associated with rheumatic affections, diabetes mellitus and albuminuria, but just how it is associated in relation to cause and effect with kidney affections, I am unable to say, but would suggest that it is due to the accumulation of effete products in the system, possibly of uric acid, urea and the like. This disorder is characterized by tumefaction of the gums, which have a purplish tint along the margins, the festoons are swollen and detached from the teeth, and bleed on the least provocation; many of the teeth are loose in their alveoli, from which pus constantly exudes, and in a majority of the cases examination of the roots of these teeth will show them to be more or less covered with calculus, the character of which is still in dispute, some claiming it is salivary, others serumal. After a little time the gums begin to recede from the necks of the teeth, and it is not by any means rare for this disease to progress so rapidly that one or more teeth are lost by the disorder before the completion of gestation. The disease usually persists after delivery, but not with such rapid progress as before. Treatment consists in removing the deposits from the affected teeth, incising the gums down to the bottom of each pocket to give drainage for the escape of the discharges, the application to the parts of a saturated solution of iodine in wood creosote, and the use of astringent and antiseptic mouth washes.

The changes in the nerious sy'stem due to the effects of pregnancy are many and varied, the character being determined largely by the individual susceptibility of the patient to nervous irritation. As a rule, the emotions are more easily excited than at other times. Some are bright and happy, others despondent, moody or peevish. Functional disorders of the special senses are not infrequent, vision may be impaired, the hearing affected, or perversions of taste and smell may occur. Neuralgic affections are quite common during pregnancy, and most frequently affect the face and head. Odontalgia, tic douleureux, local anæsthesia and paresis are the most common forms.

Odontalgia is generally the result of dental caI ries which penetrates to the pulp and sets up an 
acute or chronic pulpitis, or may be caused (as recently stated by Dr. W. W. Allport $\left.{ }^{15}\right)$, by hyperæmia of the pulp, due to the augmented volume of blood, increased arterial pressure and capillary hyperæmia of the upper balf of the body. In proof of this statement by Dr. Allport, I would call attention to the fact that a brisk cathartic will often relieve, or entirely control an attack of odontalgia, or a congestive headache, by simply relieving general arterial tension and local hyperæmia of the parts by depleting the circulation; this relieves the blood pressture upon the nerve filaments of the pulp or the meninges of the brain, and the pain ceases. Tic douloureux, local anasthesia and paresis of the facial region are generally considered to be due to a reflex neurosis, but the point made by Dr. Allport is worthy of careful consideration. Odontalgia due to pulpitis can be controlled by devitalizing the pulp, and the tooth be made permanently useful by appropriate after-treatment; a temporary stopping is all that should be attempted until after delivery if the attack occur during the latter months of gestation, but in the earlier months there need be no fear, in a majority of cases, in performing a permanent operation if it be not too fatiguing.

Nervous disorders due to the changed condition of the system during pregnancy usually disappear soon after delivery, but they occasionally persist during lactation. The treatment of neuralgic conditions of reflex origin, and nervous disorders in general, should be relegated to the family physician, as they need general treatment and therefore come more especially within his province. Quinine, iron, or other general tonic treatment, however, is indicated, with change of climatic surroundings.

The statement has been made that softening and decalcification of the bones takes place to a limited extent in certain cases of pregnancy, but whether the change is due to a failure of the nutritive processes to supply the waste constantly going on in all the tissues of the body, or an actual resorption of the inorganic materials to supply these elements to the forming foetus, I am unable to state, but am of the opinion that it is the result of both faulty nutrition and the lack of supply of the proper elements to the system. In some cases it is certainly the direct result of faulty nutrition incident to the pregnant state. In others inability to take food, as in those cases where for months the stomach is in such an irritable condition as to make it impossible to retain sufficient food to properly nourish the body; while in others it is the result of an improper diet.

Many women are in the habit of discarding from their aliment durıng pregnancy all those foods which contain an abundance of calcium salts, and restrict themselves, as nearly as possi-

"Paper on "Neuralgia," read before the American Medical Association, June, 1889 . ble, to a fruit diet, believing that by such practice the bones of the child will be imperfectly calcified, and thus parturition will be robbed of much of its suffering. There is no scientific evidence that such a result is obtained; while on the other hand, as in those cases affected with hyperemesis, though the child when born may be small and much emaciated, it has the appearance of being properly formed and its bones as dense as in the majority of normal pregnancies, and the fact seems to be pretty well established that the osseous framework of the fotus is formed in such cases at the expense of the maternal organism.

We have no knowledge that a chemical analysis has ever been made upon the teeth of the same individual before and during pregnancy in cases like those just described; and without such an analysis it could not be dogmatically asserted that there was or was not a quantitative change in the inorganic materials of the teeth, but reasoning from analogy, we would expect to find the same conditions existing in the teeth that are undoubtedly present in the bones, and brought about by the same causes.

Clinical observation would seem to substantiate such a proposition, for every observing and thoughtful dentist of even limited experience could relate cases in which a material and an ap. precrable softening of the dentine occurred during the pregnant state of many of his patients. This condition is an important predisposing cause of caries, and is often present in adults and in school children of both sexes, as a result of overwork, anxiety or excessive mental strain. ${ }^{20}$

The principal exciting cause of caries during pregnancy, as in all other cases, is the ever-present lactic acid fermentation, but its action is greatly augmented by the changed conditions of the salivary and buccal secretions. The acids found in the secretions of the mouth as a result of the disorders incident to the pregnant state, are acetic, hydrochloric, uric and oxalic. Consequently in those cases where the dentine has been rendered abnormally soft-deficient in calcium salts-there is likely to be a very rapid breaking down of tooth structure during pregnancy and lactation.

Long tedious operations, like the restoration of form in decayed teeth with gold, are inadmissible during gestation. All operations upon the teeth at such times should be as free from pain and fatigue as is possible, from the fact that in certain cases miscarriage might be the result. Caries of the teeth can be arrested by the use of temporary fillings like Hill's Stopping, or oxy-phosphate cement, until such time as the patient is in condition to bear the nervous strain incident to large gold operations. Among the most valuable of preventive measures are a thorough and frequent use of the tooth-brush and floss silk at least three

\footnotetext{
2. Jacob L. Williams, Journal Am. Med, Assoc., 1885.
} 
times per diem, supplemented by tooth powders, and antacid mouth washes.

I am aware that $I$ have not discussed this subject as its great importance would seem to deserve, and had time permitted, I should have been glad to have elaborated many points that have been only just alluded to. I trust, however, that some of these points may receive more extended treatment in the discussion that shall follow.

"The Argyle," cor. Michigan Ave. and Jackson St.

\section{DRAINAGE IN ABDOMINAL SURGERY.}

Read in the Section of Surgery and Anatomy, at the Fortieth Annual Meeting of the American Medical Association, June, r889.

BY CHARI.ES BINGHAM PENROSE, M.D., Ph.D., OF PHILADELPHIA.

Drainage of the peritoneum, though one of the most important subjects in abdominal surgery, is yet one which still stands in a most uncertain position. The reason of this uncertain position is primarily a dread of evil consequences from the tube; a fear that the tube will act as a source of septic infection and death. Other less important reasons militate.against the use of the drainage tube, but collectively they have but little weight when compared with the terror, which most surgeons feel, of introducing a dangerous element of septic infection in any case where a drainage tube is employed:

As long as this fear exists the abdominal drainage tube can never be viewed in a proper light. And it is one of my chief objects in presenting this paper, to attempt to place the subject in a more scientific position, by showing how groundless all such fears are, if the drainage tube is properly used.

But little light on this subject can be obtained by investigating the practice of noted operators. A very few operators-as Olshausen-altogether discard the abdominal drainage tube, asserting that it is in no case necessary. (Die Krankheiten der Ovarien, 1886.) Others-like Bantock and Tait-use drainage freely and with confidence, never allowing fear of evil consequences from the tube to affect their decision about its use in any case. Others-and perhaps the largest bodyoccupy a medium place; they dread the tube, but sometimes feel obliged to use it, because they dread more sepsis from absorption of undrained fluids in the peritoneum. The last hold a most unsatisfactory and a most unscientific position; and probably have the largest mortality after their operations.

If one surgeon, or class of surgeons, can use the abdominal drainage tube in a long series of cases, with a very small mortality-a mortality no greater than occurs in the same hands in a similar series of undrained cases, it certainly proves that the drainage tube properly used is not a source of danger. And it follows from this, that, if other surgeons have a much greater mor. tality when the tube is used than when it is dis. carded, the reason is that they do not employ the tube in a proper way.

The important point to observe, in the use of the abdominal drainage tube, is frequent and careful cleaning. Cleaning which can be performed by any neat nurse. If the statistics and practice of different operators are observed, it will be found that, in most cases, a large mortality and infrequent cleaning of the tube go together. We often are advised to make the first inspection of the tube not earlier than twelve hours after the operation-in some cases twentyfours hours. An investigation of cases subjected to this plan of treatment will, by the mortality, show, unquestionably, the value of frequent cleaning. I will refer to one striking illustration of the truth of this remark. There appeared recently a paper on drainage in which the writer says, "the first inspection of the tube for fluid need rarely be made earlier than twelve hours after the operation." He then gives his mortality in a series of drained cases as over 50 per cent. Compare this advice and these statissics with those of Bantock, who operated without a death on a series of 45 drained cases in the Samaritan Free Hospital, and who advises that the drainage tube be cleaned every two or three hours ; (British Medical Joumal, June 30, r888). Or with the statistics of Joseph Price, of Philadelphia, who uses drainage in about 50 per cent. of his cases of laparotomy, with a mortality as small as in the undrained cases, and who cleans the tube from every one to three hours. In my own practice, I have operated for disease of the uterus or its appendages, on 40 successive drainage cases with one death. I frequently have the tube cleaned as often as every half hour: and in introducing it, I think of it no more as a source of danger, than if I introduced an extra suture in the abdominal walls.

Many similar statistics could be given, which show that, in some hands, the drainage tube adds nothing to the mortality after laparotomy, and consequently that when it seems to increase the mortality, the cause is to be sought in the method of using the tube, and not in the tube per se.

No fixed time can be set for cleaning the abdominal drainage tube. It should always be cleaned when it is filled with fluid, and sometimes it is filled fifteen minutes after the patient has been returned to bed. By cleaning I mean the thorough emptying and drying of the tube, and the introduction of a fresh capillary drain if one is used.

Our object, after most cases of laparotomy, is to dry, as quickly and completely as possible, all the peritoneum beyond the neighborhood of the tube ; and to do this the outward flow should be 